\title{
Wnt signaling in regulation of biological functions of the nurse cell harboring Trichinella spp.
}

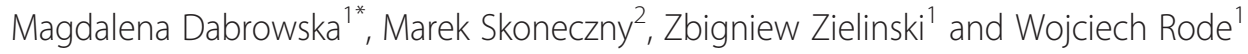

\begin{abstract}
Background: The nurse cell (NC) constitutes in mammalian skeletal muscles a confined intracellular niche to support the metabolic needs of muscle larvae of Trichinella spp. encapsulating species. The main biological functions of NC were identified as hypermitogenic growth arrest and pro-inflammatory phenotype, both inferred to depend on AP-1 (activator protein 1) transcription factor. Since those functions, as well as AP-1 activity, are known to be regulated among other pathways, also by Wnt (Wingless-Type of Mouse Mammary Tumor Virus Integration Site) signaling, transcription profiling of molecules participating in Wnt signaling cascades in NC, was performed.
\end{abstract}

Methods: Wnt signaling-involved gene expression level was measured by quantitative RT-PCR approach with the use of Qiagen $\mathrm{RT}^{2}$ Profiler PCR Arrays and complemented by that obtained by searching microarray data sets characterizing NC transcriptome.

Results: The genes involved in inhibition of canonical Wnt/ $\beta$-catenin signaling cascade as well as leading to $\beta$-catenin degradation were found expressed in NC at high level, indicating inhibition of this cascade activity. High expression in NC of genes transmitting the signal of Wnt non-canonical signaling cascades leading to activation of AP-1 transcription factor, points to predominant role of non-canonical Wnt signaling in a long term maintenance of NC biological functions.

Conclusions: Canonical Wnt/ $\beta$-catenin signaling cascade is postulated to play a role at the early stages of NC formation when muscle regeneration process is triggered. Following mis-differentiation of infected myofiber and setting of NC functional specificity, are inferred to be controlled among other pathways, by Wnt non-canonical signaling cascades.

Keywords: Trichinella spp., Nurse cell, Wnt signaling, Growth arrest, Inflammatory phenotype, AP-1 transcription factor

Abbreviations: AP-1, activator protein 1; EGF, epidermal growth factor; FGF, fibroblast growth factor; GAPDH, glyceraldehyde-3-phosphate dehydrogenase; GPCR, G protein-coupled receptor; H\&E, haematoxylin and eosin; NC, nurse cell; PCP, planar cell polarity; PDGF, platelet-derived growth factor; Wnt, Wingless-type of mouse mammary tumor virus integration site

\footnotetext{
* Correspondence: m.dabrowska@nencki.gov.pl

${ }^{1}$ Laboratory of Comparative Enzymology, Department of Biochemistry,

Nencki Institute of Experimental Biology, Polish Academy of Sciences, 3

Pasteur St., Warsaw 02-093, Poland

Full list of author information is available at the end of the article
} 


\section{Background}

The nurse cell (NC) constitutes an intracellular niche for the muscle larvae of parasitic nematode Trichinella spp. Its basic morphological structure, called cyst, is formed within mammalian striated muscles 20-28 days post-oral infection [1, 2]. Larva penetration into the muscles induces degeneration of infected myofiber, followed by its fusion with muscle satellite cells and commencement of regeneration process. However, eventually mis-differentiation takes place and part of the infected myofiber transforms into a non-muscular structure, the NC fulfilling larva metabolic requirements. NC-larva complex confined within a collagen capsule and surrounded by circulatory rete is stably maintained throughout the life span of the host [1]. NC is characterized by hypertrophy and $4 \mathrm{~N}$ DNA content $[3,4]$. Based on transcription profiling $\mathrm{NC}$ growth arrest stage was identified as being of $\mathrm{G}_{1}$-like type accompanied by cellular senescence [5]. NC was also found to display antigen presentation capability and pro-inflammatory secretory phenotype [6].

Wnt signaling pathway plays an important role in morphogenesis and postnatal stem cell fate determination $[7,8]$. Inhibition of canonical $\mathrm{Wnt} / \beta$-catenin signaling is required for cell lineage differentiation but the cascade, if recapitulated in mature differentiated cellular systems, is associated with onset of various diseases, including neurodegeneration and malignancies [9-11]. A role in cellular senescence and aging-associated disorders have been ascribed to various Wnt ligands [12-14]. Physiological responses to Wnt signaling are elicited by diverse cellular functions: cell survival, proliferation, apoptosis, differentiation, cell movement and immunological activities [15]. Wnt growth factors bind to transmembrane Frizzled (Fzd) receptors, belonging to G Protein-Coupled Receptor (GPCR) family [9]. The signal is subsequently transduced via three distinct routes: the canonical Wnt $/ \beta$-catenin and two non-canonical Wnt/ PCP (Planar Cell Polarity) and $\mathrm{Wnt} / \mathrm{Ca}^{2+}$, signaling cascades $[15,16]$. Particular Wnt ligand-Fzd receptor interactions are tissue- and process-specific. It is emphasized for Wnt signal transduction that various combinations of ligand-receptor complexes, as well as many regulatory loops and cross-talks, also with other signaling pathways, ultimately lead to a cell-specific type of response [17, 18]. Despite such a diversity, specifically Wnt 4 , Wnt 5A and Wnt 11 ligands are considered to activate Wnt noncanonical cascades $[18,19]$. Of note, Wnt 5 A upregulation was demonstrated to occur in stimulated antigenpresenting cells, i.e. dendritic cells and macrophages [20]. In the case of canonical Wnt signaling route transcription of effector genes is activated by $\beta$-catenin transcription activation complex, and in the case of non-canonical Wnt signaling route, by AP-1 transcription factor [15].
As far as skeletal muscles are concerned, Wnt signaling is involved in myogenesis and muscle regeneration. Canonical Wnt/ $/$-catenin signaling mediated by Wnt 1 and Wnt 7A ligands was shown to induce early myogenesis in mice [21]. Wnt 3A, Wnt 5A/5B and Wnt 7A/7B ligands signaling is considered critical for muscle regeneration, with myoblast differentiation and myotube fusion assumed to be affected [8]. Yet transient $\beta$-catenin activation, accompanying this process, is also viewed rather as a vestige from embryonic lineage, crucial for myogenesis but requiring inhibition for muscle regeneration to proceed [22].

As a cellular system, NC originates from muscle cells suspended during regeneration. Immunological activities with signaling pathways culminating at AP-1 transcription factor activation, were identified as its prominent biological functions [6]. Those characteristics should apparently be controlled by Wnt signaling. Additionally, Wnt 2 ligand was found in general analysis of $\mathrm{NC}$ transcriptome to be highly upregulated, in comparison to myoblastic cell line [5]. Therefore, the present scrutinized analysis was undertaken, of expression level of factors involved in Wnt signaling in NC, performed with the use of PCR arrays and supported by the search of microarray data sets [5]. The results point to a putative essential role of Wnt factors in setting of NC phenotype.

\section{Methods}

NC isolation

Trichinellosis in BALB/c mice, infected with Trichinella spiralis $\mathrm{H} 2$ human isolate, was exploited as previously described [23]. NCs were isolated from mice carrying 6 month-old infections by sequential muscle digestion, as earlier presented [5]. NC in a typical preparation is shown in Fig. 1.

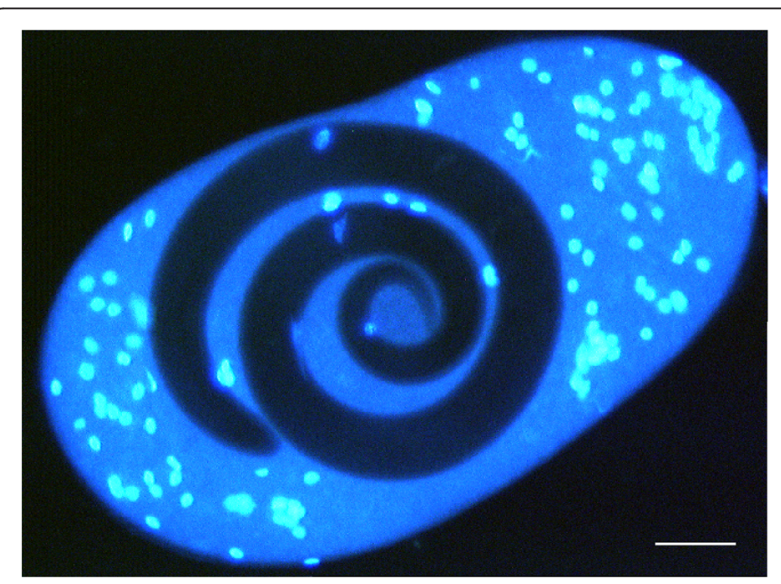

Fig. 1 NC-Trichinella spiralis larva complex. The nuclei were visualized with Hoechst 33342 dye (Lonza). The image was taken with Nikon Optiphot-Z fluorescence microscope. Scale-bar: $100 \mu \mathrm{m}$ 


\section{$\mathrm{RT}^{2}$ Profiler PCR Arrays}

Qiagen kits were used at all steps. Total RNA was isolated with the use of RNaesy Mini kit, according to manufacturer's instruction, with implementation of the RNase-Free DNase digestion step. RNA integrity was confirmed using Agilent 2100 BioAnalyzer. $\mathrm{RT}^{2}$ First Strand kit was used for reverse transcription. $\mathrm{RT}^{2} \mathrm{SYBR}$ Green/ROX qPCR Master Mix was used for quantitative PCR on Qiagen $\mathrm{RT}^{2}$ Profiler PCR Array of Mouse Wnt Signaling Pathway. The run was performed on 7500 Sequence Detection System (Applied Biosystems), including all control reactions recommended by the arrays' manufacturer. Target gene expression level was calculated according to Qiagen $\mathrm{RT}^{2}$ Profiler PCR Array handbook, applying the comparative threshold cycle $\left(C_{T}\right)$ method, with glyceraldehyde-3-phosphate dehydrogenase (GAPDH) used as a reference gene. It is given as 2exp- $\Delta C_{\mathrm{T}}$ ( \pm average deviation for $\left.n=2\right)$, where $\Delta C_{\mathrm{T}}$ is $C_{\mathrm{T}}$ (target gene) $\mathrm{C}_{\mathrm{T}}$ (GAPDH). The genes included into $\mathrm{RT}^{2}$ Profiler PCR Array whose threshold cycle fell above 35th cycle were excluded from data presentation.

\section{Microarray data sets searching}

In order to complement performed herein transcription profiling of Wnt signaling factors in $\mathrm{NC}$, the previously obtained competitive microarray data sets [5], were searched for identifiers included in Qiagen $\mathrm{RT}^{2}$ Profiler PCR Array, as well as the identifiers not included in the PCR array but otherwise related to Wnt signaling. In the aforementioned competitive microarray analysis, the transcriptomes of $\mathrm{C} 2 \mathrm{C} 12$ myoblasts and $\mathrm{C} 2 \mathrm{C} 12$ myotubes served as referral systems to the NC transcriptome. In order to eliminate biological differences among NC preparations, four different preparations of NCs isolated from mice carrying 5 to 12 month-infections were exploited for competitive microarray analysis. Only the identifiers with differential gene expression level $\geq 2$ accompanied by a $P$-value $\leq 0.05$, were considered signaling pathway-eligible. Fold change in gene expression level in $\mathrm{NC}$, in relation to $\mathrm{C} 2 \mathrm{C} 12$ myoblasts or myotubes, was calculated form $\log _{2}$ ratio value and is provided as the average of quadruplicates, accompanied by the $P$-values calculated by Student's one-sample $t$-test. All parameters of statistical analysis, including $\log _{2}$ ratio \pm standard deviation (SD) as well as the $t$-values, are shown in Additional file 1: Table S1.

\section{Results and discussion}

\section{Characteristics of NC formation process}

The NC is a non-muscular structure originating from a few types of cells. During encapsulation of the larva lasting up to 28 days post-infection, the nuclei, mitochondria and basophilic cytoplasm (i.e. staining with haematoxylin in haematoxylin and eosin (H\&E) staining protocol), of infected myofiber, degenerate with the signs of apoptosis and autocrine signaling by tumor necrosis factor $\alpha$ [24, 25]. Inhibition of transforming growth factor $\beta$ signaling by c-Ski repressor was also shown to accompany this process [26]. Muscle satellite cells, fusing with the infected degenerating myofiber, become the main source of nuclei, mitochondria and eosinophilic cytoplasm (i.e. staining with eosin in H\&E staining procedure), in the completely established $\mathrm{NC}$ at 3month-old infection. Some nuclei of NC become hypertrophied at this stage, and infiltrating lymphocytes were also identified entrapped in the NC cytoplasm [27]. It should be noted that during $\mathrm{NC}$ formation two various kinds of cytoplasm, basophilic and eosinophilic, are separated by plasma membrane and the whole process in independent on p53 suppressor gene [2, 27, 28]. Analysis of NC transcriptome during the process of intracellular transformation (i.e. 23rd day post-infection), indicated activation of survival mechanism mediated by insulin-like growth factor 1 which may lead to induction of AP-1 transcription factor $[29,30]$. Wht $8 \mathrm{~A}$ and $5 \mathrm{~B}$ ligand expression was also found upregulated at this stage of NC development, as analyzed in the whole infected $v s$ uninfected muscle tissue [29]. Wnt canonical signaling cascade inhibitory factor Dickkopf homolog 4 (DKK4 gene) [31], was also found upregulated in those settings [29]. These findings indicate that already at the stage of larva encapsulation Wnt signaling-involved factors shape NC functional specificity towards noncanonical Wnt signaling and AP-1 factor activation, serving to determine survival and immunological properties.

\section{Inhibitory factors of canonical Wnt/ $\beta$-catenin signaling cascade are expressed in fully established NC}

A network of molecules participating in Wnt signaling, whose expression was detected in NC, is depicted in Fig. 2. Gene description and gene expression levels are shown in Table 1 . In cells unstimulated by Wnt ligands, central molecule of this cascade, $\beta$-catenin (encoded by CTNNB1 gene), is known to remain in the cytoplasm in a phosphorylated form complexed with GSK3B and scaffolding factors APC and AXIN1 [32]. Apart from GSK3B, also casein kinases, represented in $\mathrm{NC}$ by CSNK1A1, CSNK1D and CSNK2A1, are known to phosphorylate $\beta$-catenin. Additionally, bound with protein phosphatases (PPP2CA, PPP2R1A and PPP2R5D subunits are expressed in $\mathrm{NC}$ ), $\beta$-catenin is ubiqutinated in the presence of BTRC and driven for proteasomal degradation [32]. SENP2 peptidase is also known to participate in downregulation of $\beta$-catenin level $[33,34]$. Assuming autocrine stimulation to occur, the $\mathrm{Wnt} / \beta$-catenin signaling cascade can be activated in NC by Wnt 1 , Wnt 2/2B, Wnt 3/3A, Wnt 6, Wnt 9A and Wnt 16. Upon 


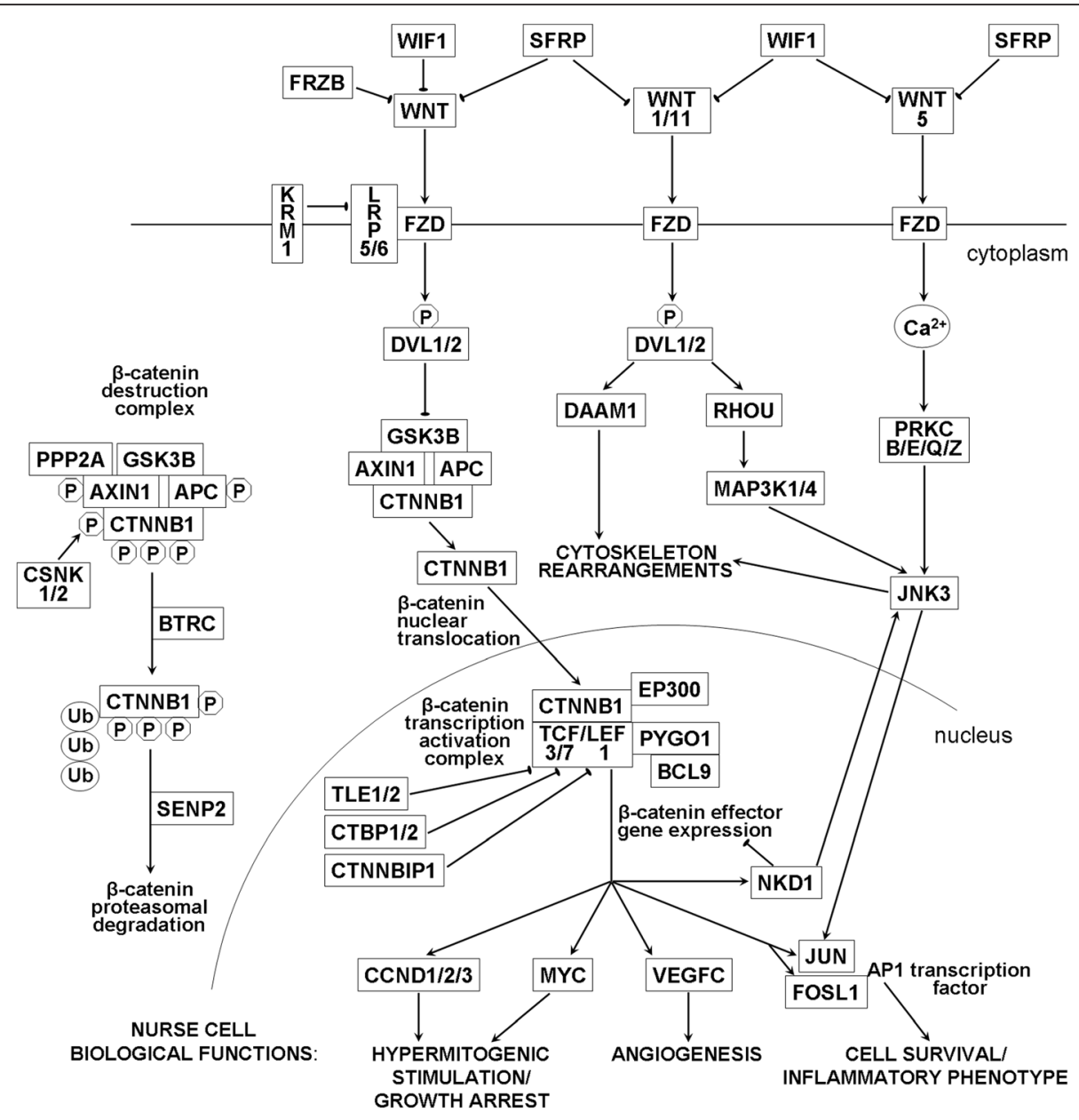

Fig. 2 Summary of interactions involved in Wnt signaling cascades inferred to regulate NC biological functions. Only the molecules whose expression was detected in NC by RT ${ }^{2}$ Profiler PCR Arrays and/or microarrays, are marked. Wnt signaling consensus pathway was complied from Wnt signaling pathway available at www.qiagen.com/pl/shop/genes-and-pathways/pathway-details/?pwid=474, Ingenuity Pathway Analysis software (www.ingenuity.com/products.ipa) and references [9, 15]. Sharp arrows indicate activatory interactions and blunt arrows indicate inhibitory interactions. Descriptions of molecule names are given in Table 1

stimulation of FZD receptors by Wnt ligands activated Dsh1/2 proteins (encoded by DVL1/2 genes), lead to inhibition of $\beta$-catenin phosphorylation. Unphosphorylated $\beta$-catenin translocates to the nucleus where it forms a transcription activation complex with TCF/LEF factors, additionally activated by EP300 and BCL9/PYGO1 complex [32]. FZD receptors 1 through 8, coreceptors LRP5/6, as well as DVL1/2, TCF3/7 and LEF1 genes, are expressed in NC, though TCF7 and LEF1 are down regulated in relation to $\mathrm{C} 2 \mathrm{C} 12$ cellular systems. Numerous molecules known to inhibit $\beta$-catenin transcription activation complex, including TLE1/2, CTBP1/2, CTNNBIP1, as well as an effector, and simultaneously an inhibitor, of this cascade, NKD1 gene product [35], are expressed in NC. NKD1 expression is also very highly upregulated in relation to $\mathrm{C} 2 \mathrm{C} 12$ myoblasts/myotubes. Of note, NKD1 is known to switch Wnt signaling from canonical to noncanonical Wnt/PCP cascade [36]. Apart from apparent inhibition of canonical Wnt/ $\beta$-catenin signaling cascade in $\mathrm{NC}$ by intracellular factors, this cascade may also be inhibited at the plasma membrane level. KRM1 (alias KREMEN1), WIF1, FRZB (alias SFRP3) and SFRP1/2/4 gene products, expressed in $\mathrm{NC}$, are known to inhibit Wnt signaling via interaction with LRP5/6 coreceptors (KRM1), binding to Wnt ligands (WIF1 and FRZB) or interaction with both Wnt ligands and Fzd receptors (SFRP1/2/4 factors) [37, 38]. Importance of an inhibitory route of canonical Wnt/ $\beta$-catenin signaling in $\mathrm{NC}$ at the plasma membrane level is further stressed by very high upregulation of FRZB and high upregulation of WIF1 expression in $\mathrm{NC}$, in relation to $\mathrm{C} 2 \mathrm{C} 12$ myoblasts/myotubes. Of note is that Wnt 1 inducible signaling pathway protein 1 WISP1, known to display anti-apoptotic activity [39], is expressed in $\mathrm{NC}$ at the level significantly lower than in $\mathrm{C} 2 \mathrm{C} 12$ cellular systems. Effector genes of canonical Wnt/ $\mathrm{\beta}$ catenin signaling cascade expressed in $\mathrm{NC}$ include factors 
Table 1 Expression in NC of molecules involved in Wnt signaling pathway. Gene expression level determined by RT ${ }^{2}$ Profiler PCR Arrays is given as $2^{-\Delta C T}$, and by competitive microarray approach as a fold change, increase or decrease $(\downarrow)$ in relation to C2C12 myoblasts and myotubes. Data analysis was performed as described under Methods section

\begin{tabular}{|c|c|c|c|c|}
\hline $\begin{array}{l}\text { GenBank accession } \\
\text { number }\end{array}$ & Gene symbol & Description & $\begin{array}{l}\text { Gene expression } 2^{-\Delta C T} \\
( \pm A D, n=2)\end{array}$ & $\begin{array}{l}\text { Fold change ( } P \text {-value) in gene } \\
\text { expression level in NC vs C2C12 } \\
\text { Myoblasts/myotubes }\end{array}$ \\
\hline NM_007462 & APC & Adenomatous polyposis coli & $2.92 \pm 0.40$ & \\
\hline NM_009733 & AXIN1 & Axin1 & $0.14 \pm 0.06$ & \\
\hline NM_029933 & BCL9 & B-cell CLL/lymphoma 9 & $0.16 \pm 0.03$ & \\
\hline NM_009771 & BTRC & Beta-transducin repeat containing protein & $0.63 \pm 0.04$ & \\
\hline NM_023465 & CTNNBIP1 & Catenin beta interacting protein 1 & $1.59 \pm 0.01$ & $/ 2.1(0.00)$ \\
\hline NM_007631 & CCND1 & Cyclin D1 & $4.40 \pm 0.62$ & $19.5(0.00)^{*}$ \\
\hline NM_009829 & CCND2 & Cyclin D2 & $19.33 \pm 1.41$ & $5.1(0.00)^{*} / 7.8(0.00)^{*}$ \\
\hline NM_007632 & CCND3 & Cyclin D3 & $2.37 \pm 0.64$ & $2.1(0.01) / \downarrow 1.9(0.01)$ \\
\hline NM_146087 & CSNK1A1 & Casein kinase 1, alpha 1 & $3.85 \pm 1.01$ & $/ \downarrow 2.1(0.00)$ \\
\hline NM_139059 & CSNK1D & Casein kinase 1 , delta & $7.36 \pm 1.33$ & \\
\hline NM_007788 & CSNK2A1 & Casein kinase 2, alpha 1 polypeptide & $9.55 \pm 0.80$ & $\downarrow 2.9(0.00) / \downarrow 3.4(0.00)$ \\
\hline NM_013502 & СТBP1 & C-terminal binding protein 1 & $0.38 \pm 0.12$ & \\
\hline NM_009980 & СТВP2 & C-terminal binding protein 2 & $5.26 \pm 0.17$ & /2.2(0.01) \\
\hline NM_007614 & CTNNB1 & Catenin (cadherin associated protein), beta 1 & $2.03 \pm 0.40$ & \\
\hline NM_172464 & DAAM1 & Dishevelled associated activator of morphogenesis 1 & $5.13 \pm 0.47$ & \\
\hline NM_010091 & DVL1 & Dishevelled 1, dsh homolog (Drosophila) & $0.48 \pm 0.13$ & $/ \downarrow 2.2(0.00)$ \\
\hline NM_007888 & DVL2 & Dishevelled 2, dsh homolog (Drosophila) & $0.09 \pm 0.04$ & \\
\hline NM_177821 & EP300 & E1A binding protein $\mathrm{p} 300$ & $0.22 \pm 0.06$ & \\
\hline NM_010234 & FOS & v-FOS murine viral oncogene homolog & & $116.7(0.00)^{*} / 27.2(0.00)^{*}$ \\
\hline NM_008036 & FOSB & FBJ murine viral oncogene homolog & & $6.0(0.00)^{*} / 5.5(0.00)^{*}$ \\
\hline NM_010235 & FOSL1 & Fos-like antigen 1 & $1.36 \pm 0.13$ & $\downarrow 3.1(0.00) / 2.1(0.01)$ \\
\hline NM_011356 & FRZB & Frizzled-related protein & $8.25 \pm 2.13$ & $21.9(0.00) / 14.8(0.00)$ \\
\hline NM_021457 & FZD1 & Frizzled homolog 1 (Drosophila) & $1.36 \pm 0.46$ & $3.6(0.00) /$ \\
\hline NM_020510 & FZD2 & Frizzled homolog 2 (Drosophila) & $1.20 \pm 0.77$ & \\
\hline NM_021458 & FZD3 & Frizzled homolog 3 (Drosophila) & $0.39 \pm 0.04$ & \\
\hline NM_008055 & FZD4 & Frizzled homolog 4 (Drosophila) & $0.35 \pm 0.00$ & $3.6(0.01) /$ \\
\hline NM_022721 & FZD5 & Frizzled homolog 5 (Drosophila) & $1.97 \pm 0.26$ & \\
\hline NM_008056 & FZD6 & Frizzled homolog 6 (Drosophila) & $0.22 \pm 0.11$ & \\
\hline NM_008057 & FZD7 & Frizzled homolog 7 (Drosophila) & $0.07 \pm 0.04$ & \\
\hline NM_008058 & FZD8 & Frizzled homolog 8 (Drosophila) & $0.02 \pm 0.01$ & $3.4(0.00) / 2.4(0.03)$ \\
\hline NM_019827 & GSK3B & Glycogen synthase kinase 3 beta & $0.75 \pm 0.02$ & \\
\hline NM_010591 & JUN & Jun oncogene & $7.68 \pm 2.99$ & $1.9(0.00) /$ \\
\hline NM_010592 & JUND & Jun-D proto-oncogene & & $2.5(0.02)^{*} / 2.1(0.02)^{*}$ \\
\hline NM_032396 & KREMEN1 & Kringle containing transmembrane protein 1 & $8.56 \pm 0.19$ & \\
\hline NM_010703 & LEF1 & Lymphoid enhancer binding factor 1 & $0.01 \pm 0.003$ & $\downarrow 3.7(0.00) / \downarrow 2.4(0.04)$ \\
\hline NM_008513 & LRP5 & Low density lipoprotein receptor-related protein 5 & $1.43 \pm 0.63$ & $3.4(0.00) / 3.9(0.00)$ \\
\hline NM_008514 & LRP6 & Low density lipoprotein receptor-related protein 6 & $1.44 \pm 0.09$ & \\
\hline NM_011945 & MAP3K1 & MEKK1, MAP kinase kinase kinase 1 & & $4.9(0.00)^{*} / 6.7(0.00)^{*}$ \\
\hline NM_011948 & MAP3K4 & MEKK4, MAP kinase kinase kinase 4 & & $2.1(0.00)^{*} / 2.0(0.00)$ \\
\hline NM_009158 & MAPK10 & JNK3, Jun-N terminal kinase & & $8.8(0.00)^{*} / 6.8(0.00)^{*}$ \\
\hline NM_010849 & MYC & Myelocytomatosis oncogene & $0.88 \pm 0.14$ & $\downarrow 4.7(0.00)^{*} / \downarrow 2.9(0.01)^{*}$ \\
\hline
\end{tabular}


Table 1 Expression in NC of molecules involved in Wnt signaling pathway. Gene expression level determined by RT ${ }^{2}$ Profiler PCR Arrays is given as $2^{-\Delta C T}$, and by competitive microarray approach as a fold change, increase or decrease $(\downarrow)$ in relation to C2C12 myoblasts and myotubes. Data analysis was performed as described under Methods section (Continued)

\begin{tabular}{|c|c|c|c|c|}
\hline NM_027280 & NKD1 & Naked cuticle 1 homolog (Drosophila) & $0.37 \pm 0.04$ & $42.3(0.00) / 27.8(0.00)$ \\
\hline NM_008702 & NLK & Nemo-like kinase & $0.38 \pm 0.01$ & \\
\hline NM_019411 & PPP2CA & $\begin{array}{l}\text { Protein phosphatase } 2 \text { (formerly } 2 \mathrm{~A} \text { ), catalytic subunit, } \\
\text { alpha isoform }\end{array}$ & $15.79 \pm 0.81$ & \\
\hline NM_016891 & PPP2R1A & $\begin{array}{l}\text { Protein phosphatase } 2 \text { (formerly 2A), regulatory subunit } \\
\text { A (PR 65), alpha isoform }\end{array}$ & $10.09 \pm 0.04$ & \\
\hline NM_009358 & PPP2R5D & $\begin{array}{l}\text { Protein phosphatase } 2 \text {, regulatory subunit B (B56), delta } \\
\text { isoform }\end{array}$ & $0.75 \pm 0.14$ & \\
\hline NM_008855 & PRKCB1 & Protein kinase $\mathrm{C}$, beta 1 & & $10.0(0.01)^{*} / 6.7(0.00)^{*}$ \\
\hline AK017901 & PRKCE & Protein kinase $C$, epsilon & & $4.3(0.01)^{*} / 4.4(0.00)^{*}$ \\
\hline NM_008859 & PRKCQ & Protein kinase $C$, theta & & $6.3(0.00)^{*} / 4.6(0.01)^{*}$ \\
\hline NM_008860 & PRKCZ & Protein kinase C, zeta & & $14.6(0.00)^{*} / 10.6(0.00)^{*}$ \\
\hline NM_028116 & PYGO1 & Pygopus 1 & $0.38 \pm 0.01$ & $4.6(0.00) / 3.0(0.01)$ \\
\hline NM_133955 & $\mathrm{RHOU}$ & Ras homolog gene family, member $U$ & $0.51 \pm 0.16$ & $/ 2.3(0.00)$ \\
\hline NM_029457 & SENP2 & SUMO/sentrin specific peptidase 2 & $1.88 \pm 0.05$ & \\
\hline NM_013834 & SFRP1 & Secreted frizzled-related protein 1 & $0.09 \pm 0.008$ & \\
\hline NM_009144 & SFRP2 & Secreted frizzled-related protein 2 & $0.02 \pm 0.012$ & $/ \downarrow 5.4(0.00)$ \\
\hline NM_016687 & SFRP4 & Secreted frizzled-related protein 4 & $0.01 \pm 0.003$ & \\
\hline NM_009332 & TCF3 & Transcription factor 7-like 1 (T-cell specific, HMG box) & $1.31 \pm 0.10$ & $2.1(0.02) / 2.6(0.00)$ \\
\hline NM_009331 & TCF7 & Transcription factor 7, T-cell specific & $0.46 \pm 0.13$ & $\downarrow 3.2(0.00) / \downarrow 2.3(0.01)$ \\
\hline NM_011599 & TLE1 & Transducin-like enhancer of split 1 & $1.10 \pm 0.27$ & \\
\hline NM_019725 & TLE2 & Transducin-like enhancer of split 2 & $0.005 \pm 0.0022$ & \\
\hline NM_009506 & VEGFC & Vascular endothelial growth factor $C$ & & $5.3(0.00)^{*} / 5.9(0.00)^{*}$ \\
\hline NM_011915 & WIF1 & Wnt inhibitory factor 1 & $0.15 \pm 0.02$ & $6.4(0.00) / 2.5(0.00)$ \\
\hline NM_018865 & WISP1 & WNT1 inducible signaling pathway protein 1 & $2.22 \pm 0.69$ & $\downarrow 4.9(0.00) / \downarrow 6.1(0.00)$ \\
\hline NM_021279 & WNT1 & Wingless-related MMTV integration site 1 & $0.003 \pm 0.0011$ & \\
\hline NM_009519 & WNT11 & Wingless-related MMTV integration site 11 & $1.89 \pm 0.22$ & $6.5(0.00) / 4.1(0.00)$ \\
\hline NM_053116 & WNT16 & Wingless-related MMTV integration site 16 & $0.14 \pm 0.01$ & $5.6(0.01) / 3.3(0.00)$ \\
\hline NM_023653 & WNT2 & Wingless-related MMTV integration site 2 & $0.008 \pm 0.0029$ & $28.2(0.00)^{*} / 21.9(0.00)^{*}$ \\
\hline NM_009520 & WNT2B & Wingless related MMTV integration site $2 \mathrm{~b}$ & $0.03 \pm 0.001$ & \\
\hline NM_009521 & WNT3 & Wingless-related MMTV integration site 3 & $0.002 \pm 0.0013$ & $3.3(0.01) / 2.4(0.00)$ \\
\hline NM_009522 & WNT3A & Wingless-related MMTV integration site $3 \mathrm{~A}$ & $0.004 \pm 0.0022$ & \\
\hline NM_009523 & WNT4 & Wingless-related MMTV integration site 4 & $0.009 \pm 0.0062$ & $\downarrow 2.9(0.00) /$ \\
\hline NM_009524 & WNT5A & Wingless-related MMTV integration site $5 \mathrm{~A}$ & $0.03 \pm 0.011$ & $4.8(0.01) / 3.0(0.02)$ \\
\hline NM_009525 & WNT5B & Wingless-related MMTV integration site $5 B$ & $5.89 \pm 2.90$ & $9.9(0.00) / 7.7(0.00)$ \\
\hline NM_009526 & WNT6 & Wingless-related MMTV integration site 6 & $0.03 \pm 0.008$ & $\downarrow 2.8(0.01) /$ \\
\hline NM_139298 & WNT9A & Wingless-type MMTV integration site 9A & $0.01 \pm 0.005$ & $2.5(0.00) / \downarrow 2.1(0.00)$ \\
\hline
\end{tabular}

*Asterisks mark gene expression level, determined by microarray approach and reported previously in the context of other signaling pathway analyses $[5,6]$

involved in regulation of cell fate and inflammation: cyclins D, c-Myc, Fra1 (encoded by FOSL gene) and c-Jun, as well as angiogenic factor VEGFC [web.stanford.edu/group/ nusselab/cgi-bin/wnt/target_genes].

It is thus inferred that dominant expression in $\mathrm{NC}$ of molecules involved in $\beta$-catenin degradation as well as inhibition of canonical Wnt signal transduction and $\beta$ - catenin-dependent transcription, indicate that even though could be operating, Wnt $/ \beta$-catenin signaling cascade is inhibited. Expression of the cascade effector genes may have resulted from $\mathrm{Wnt} / \beta$-catenin-activated transcription at the earlier stages of NC formation, but in fully established $\mathrm{NC}$ this regulation seems to be attributed rather to other signaling pathways. 
Effector factors of non-canonical Wnt/PCP and Wnt/Ca ${ }^{2+}$ signaling cascades are expressed in fully established NC A network of molecules participating in non-canonical Wnt signaling cascades, whose expression was detected in $\mathrm{NC}$, is schematically depicted in Fig. 2, with gene descriptions and expression level values provided in Table 1. Non-canonical Wnt signaling was shown in various cellular systems to be stimulated by Wnt $1 / 11$ and Wnt 5A ligands [15, 40, 41]. Wnt 11 and Wnt 5B are expressed in $\mathrm{NC}$ at the highest level among other Wnt ligands. Their expression, as well as the expression of Wnt $5 \mathrm{~A}$, is also upregulated in relation to $\mathrm{C} 2 \mathrm{C} 12$ myoblasts/myotubes. Similar to the canonical cascade, activation of Wnt/PCP cascade occurs via phosphorylation of Dsh proteins [16]. In NC, the signal can be transduced downstream by DAAM1 factor and RHOUMAP3K1/4-JNK3 axis, to induce cytoskeleton rearrangements. JNK3 can also be activated in $\mathrm{NC}$ via $\mathrm{Ca}^{2+}$ and protein kinase $\mathrm{C}$ axis, known to be activated also by classical GPCRs. JNK3 phosphorylates c-Jun and JunD which then dimerise with one of the Fos proteins to form transcription factor AP-1, known to display prosurvival and proinflammatory action, as well as to inhibit myogenesis [16, 42-47]. Expression of c-Jun, JunD, Fra-1 (encoded by FOSL1 gene), FosB and Fos is found in NC, with Fos being the most highly upregulated gene in relation to $\mathrm{C} 2 \mathrm{C} 12$ cellular systems. Thus expression in $\mathrm{NC}$ of Wnt 11, Wnt 5A/5B ligands, as well as JNK3 and Jun/Fos factors, indicate importance of AP-1 factor in maintenance of NC biological functions mediated by noncanonical Wnt signaling cascades. One of the effector genes of $\mathrm{Wnt} / \mathrm{Ca}^{2+}$ signaling cascade, expressed in $\mathrm{NC}$, is Nemo-like kinase (NLK, Table 1). As NLK is known to suppress $\beta$-catenin-dependent transcription [48], its expression in $\mathrm{NC}$ further points to inhibition of canonical Wnt signaling cascade.

It is inferred from the study performed that canonical, as well as non-canonical cascades operate in $\mathrm{NC}$ at the various stages of its formation. Expression in the fully established NC of Wnt ligands responsible for activation of canonical Wnt signaling cascade, the latter known to accompany induction of muscle regeneration $[8,22]$, may reflect the vestiges from those stages of NC formation when muscle regeneration was triggered. Eventually the cascade inhibition prevails. It is also possible that expression in fully established $\mathrm{NC}$ of the cascade inhibiting factors, including a feedback inhibitor NKD1, is indicative of execution of a tight control of the remaining activity of canonical Wnt signaling. It can be hypothesized that at the time point of larva penetration Wnt autocrine signaling may be responsible for $\beta$-catenin-dependent induction of infected myofiber regeneration. As no differentiation ultimately occurs, probably due to influence of EGF (epidermal growth factor)/FGF (fibroblast growth
factor)/PDGF (platelet-derived growth factor)- induced proliferative stimulation [5], Wnt 5A/5B- and Wnt 11activated non-canonical signaling cascades sustain the activation of AP-1 transcription factor to regulate NC growth arrest and immunological functions [6]. Current analysis was based on putative loops of autocrine signaling operating in NC. Parasite-derived factors and paracrine signaling should also control $\mathrm{NC}$ formation and the functioning of $\mathrm{NC}$ at fully established stage. Long-term maintenance of $\mathrm{NC}$ biological specificity apparently results from a precise orchestration of various cellular signaling events. The nature of the exact factor causing transformation of muscular cells to the parasite-favorable environment, remains to be identified.

\section{Conclusions}

The NC is an intracellular habitat for Trichinella spp. muscle larvae. Assuming autocrine signaling by Wnt ligands to occur during a long-term existence of the NC-Trichinella muscle larva complex, the canonical Wnt signaling cascade is inferred to be inhibited, but the non-canonical Wnt/PCP and $\mathrm{Wnt} / \mathrm{Ca}^{2+}$ cascades are postulated to lead to maintenance of AP-1 transcription factor activation and execution of $\mathrm{NC}$ biological functions.

\section{Additional file}

Additional file 1: Table S1. The parameters of statistical analysis of competitive expression microarray data showing gene expression level in $\mathrm{NC}$ related to either $\mathrm{C} 2 \mathrm{C} 12$ myoblasts or myotubes. Only the genes referred to in Table 1 and Fig. 2 of the main body of the publication, are shown. (DOC $70 \mathrm{~kb}$ )

\section{Acknowledgements}

Not applicable.

\section{Funding}

This study was supported by National Science Center grant no. 2011/01/B/ NZ6/01781.

Availability of data and materials

The datasets supporting the conclusions of this article are included within the article and Additional file 1.

\section{Authors' contributions}

MD performed NC isolation, PCR arrays and pathway analysis. MS performed microarray data analysis. ZZ carried out parasite culture. WR coordinated implementation of the project. All authors read and approved the final version of the manuscript.

\section{Competing interests}

The authors declare that they have no competing interests.

Consent for publication

Not applicable.

Ethics approval and consent to participate

Ethical approval for this study was granted by the First Warsaw Local Ethics Committee for Animal Experimentation at the Nencki Institute. 


\section{Author details}

Laboratory of Comparative Enzymology, Department of Biochemistry, Nencki Institute of Experimental Biology, Polish Academy of Sciences, 3 Pasteur St., Warsaw 02-093, Poland. ²Department of Genetics, Institute of Biochemistry and Biophysics, Polish Academy of Sciences, 5A Pawinskiego St., Warsaw 02-106, Poland.

Received: 28 June 2016 Accepted: 22 August 2016

Published online: 02 September 2016

\section{References}

1. Despommier DD. How does Trichinella spiralis make itself at home? Parasitol Today. 1998;14:318-23.

2. Boonmars T, Wu Z, Nagano I, Takahashi Y. What is the role of p53 during the cyst formation of Trichinella spiralis? A comparable study between knockout mice and wild type mouse. Parasitology. 2005;131:705-12.

3. Jasmer DP. Trichinella spiralis infected muscle cells arrest in $\mathrm{G}_{2} / \mathrm{M}$ and cease muscle gene expression. J Cell Biol. 1993;121:785-93.

4. Jasmer DP. Trichinella spiralis: subversion of differentiated mammalian skeletal muscle cells. Parasitol Today. 1995;11:185-8.

5. Dabrowska M, Skoneczny M, Zielinski Z, Rode W. Nurse cell of Trichinella spp. as a model of long-term cell cycle arrest. Cell Cycle. 2008;7:2167-78.

6. Dabrowska M. Inflammatory phenotype of the nurse cell harboring Trichinella spp. Vet Parasitol. 2013:194:150-4

7. van Amerongen $R$, Nusse R. Towards an integrated view of Wnt signaling in development. Development. 2009:136:3205-14.

8. Tsivitse S. Notch and Wnt signaling, physiological stimuli and postnatal myogenesis. Int J Biol Sci. 2010;6:268-81.

9. Anastas JN, Moon RT. WNT signaling pathways as therapeutic targets in cancer. Nat Rev Cancer. 2013;13:11-26.

10. Powers S, Mu D. Genetic similarities between organogenesis and tumorigenesis of the lung. Cell Cycle. 2008;2:200-4

11. Verani R, Cappuccio I, Spinsanti P, Gradini R, Caruso A, Magnotti MC Expression of the Wnt inhibitor Dickkopf-1 is required for the induction of neural markers in mouse embryonic stem cells differentiating in response to retinoic acid. J Neurochem. 2007;100:242-50.

12. Ye X, Zerlanko B, Kennedy A, Banumathy G, Zhang R, Adams PD. Downregulation of Wnt signaling is a trigger for formation of facultative heterochromatin and onset of cell senescence in primary human cells. Mol Cell. 2007;27:183-96.

13. Liu H, Fergusson MM, Castilho RM, Liu J, Cao L, Chen J, et al. Augmented Wnt signaling in a mammalian model of accelerated aging. Science. 2007;317:803-6.

14. Brack AS, Conboy MJ, Roy S, Lee M, Kuo CJ, Keller C, Rando TA. Increased Wht signaling during aging alters muscle stem cell fate and increases fibrosis. Science. 2007:317:807-10.

15. Staal FJT, Tiago CL, Tiemessen MM. WNT signaling in the immune system: WNT is spreading its wings. Nat Rev Immunol. 2008:8:581-93.

16. Lai S-L, Chien AJ, Moon RT. Wnt/Fzd signaling and the cytoskeleton: potential roles in tumorigenesis. Cell Res. 2009:19:532-45.

17. Niehrs C. The complex world of WNT receptor signaling. Nat Rev Mol Cell Biol. 2012;13:767-79.

18. Nusse R. Wnt signaling in disease and in development. Cell Res. 2005;15:28-32

19. Schlessinger K, Hall A, Tolwinski N. Wht signaling pathways meet Rho GTPases. Genes Dev, 2009:23:265-77.

20. Lehtonen A, Ahlfors $H$, Veckman V, Miettinen M, Lahesmaa R, Julkunen I. Gene expression profiling during differentiation of human monocytes to macrophages or dendritic cells. J Leukoc Biol. 2007;82:710-20.

21. Bryson-Richardson RJ, Currie PD. The genetics of vertebrate myogenesis. Nat Rev Genet. 2008;9:632-46.

22. Murphy MM, Keefe AC, Lawson JA, Flygare SD, Yandell M, Kardon G. Transiently active $W n t / \beta$-catenin signaling is not required but must be silenced for stem cell function during muscle regeneration. Stem Cell Rep. 2014:3:475-88.

23. Dabrowska M, Zielinski Z, Wranicz M, Michalski R, Pawelczak K, Rode W. Trichinella spiralis thymidylate synthase: developmental pattern, isolation, molecular properties, and inhibition by substrate and cofactor analogues. Biochem Biophys Res Commun. 1996:228:440-5.

24. Boonmars T, Wu Z, Nagano I, Takahashi Y. Expression of apoptosis-related factors in muscles infected with Trichinella spiralis. Parasitology. 2004; 128:323-32
25. Wu Z, Nagano I, Boonmars T, Takahashi Y. Tumor necrosis factor receptormediated apoptosis in Trichinella spiralis-infected muscle cells. Parasitology. 2005;131:373-81.

26. Wu Z, Nagano I, Boonmars T, Takahashi Y. Involvement of the c-Ski oncoprotein in cell cycle arrest and transformation during nurse cel formation after Trichinella spiralis infection. Int J Parasitol. 2006;36:1159-66.

27. Matsuo A, Wu Z, Nagano I, Takahashi Y. Five types of nuclei present in the capsule of Trichinella spiralis. Parasitology. 2000;121:203-10.

28. Wu Z, Matsuo A, Nakada T, Nagano I, Takahashi Y. Different response of satellite cells in the kinetics of myogenic regulatory factors and ultrastructural pathology after Trichinella spiralis and T. pseudospiralis infection. Parasitology. 2001;123:85-94.

29. Wu Z, Nagano I, Boonmars T, Takahashi Y. A spectrum of functional genes mobilized after Trichinella spiralis infection in skeletal muscle. Parasitology. 2005;130:561-73.

30. Wu Z, Sofronic-Milosavljevic L, Nagano I, Takahashi Y. Trichinella spiralis: nurse cell formation with emphasis on analogy to muscle cell repair. Parasit Vectors. 2008;1:27.

31. Zorn AM. Wnt signaling: Antagonistic Dickkopfs. Curr Biol. 2001;11:R592-5.

32. Mosimann C, Hausmann G, Baster K. B-catenin hits chromatin: regulation of Wnt target gene activation. Nat Rev Mol Cell Biol. 2009;10:276-86.

33. Kadoya T, Kishida S, Fukui A, Hinoi T, Michiue T, Asashima M, Kikuchi A. Inhibition of Wnt signaling pathway by a novel axin-binding protein. J Biol Chem. 2000:275:37030-7.

34. Nishida T, Kanedo F, Kitagawa M, Yasuda H. Characterization of a novel mammalian SUMO-1/Smt3-specific isopeptidase, a homologue of rat axam, which is an axin-binding protein promoting $\beta$-catenin degradation. J Biol Chem. 2001;276:39060-6.

35. Angonin D, Van Raay TJ. Nkd1 functions as a passive antagonist of Wht signaling. PLoS One. 2013;8:e74666.

36. Katoh M. WNT/PCP signaling pathway and human cancer. Oncol Rep. 2005;14:1583-8.

37. Clevers $\mathrm{H}$, Nusse R. Wnt/ß-catenin signaling and disease. Cell. 2012;149:1192-205.

38. Mao B, Wu W, Davidson G, Marhold J, Li M, Melcher BM, et al. Kremen proteins are Dickkopf receptors that regulate $\mathrm{Wnt} / \beta$-catenin signaling. Nature. 2002:417:664-7.

39. Su F, Overholtzer M, Besser D, Levine AJ. WISP-1 attenuates p53-mediated apoptosis in response to DNA damage through activation of the Akt kinase. Genes Dev. 2002;16:46-57.

40. Tao E, Pennica D, Xu L, Kalejta RF, Levine AJ. Wrch-1, a novel member of the Rho gene family that is regulated by Wnt-1. Genes Dev. 2001;15:1796-807.

41. De A. Wnt/Ca ${ }^{2+}$ signaling pathway: a brief overview. Acta Biochim Biophys Sin. 2011:43:754-6.

42. Conejo R, Valverde AM, Banito M, Lorenzo M. Insulin produces myogenesis in C2C12 myoblasts by induction of NF-kB and downregulation of AP1 activities. J Cell Physiol. 2001;186:82-94

43. Johnson GL, Nakamura K. The c-Jun kinase/stress-activated pathway: regulation, function and role in human disease. Biochim Biophys Acta. 2007:1773:1341-8.

44. Kallunki T, Deng T, Hibi M, Karin M. c-Jun can recruit JNK to phosphorylate dimerization partners via specific docking interactions. Cell. 1996;87:929-39.

45. Li M-D, Yang X. A retrospective on nuclear receptor regulation of inflammation: lessons from GR and PPARs. PPAR Res. 2011;2011:742785.

46. Newton K, Dixit VM. Signaling in innate immunity and inflammation. Cold Spring Harb Perspect Biol. 2012;4:a006049.

47. Park K, Chung M, Kim S-J. Inhibition of myogenesis by okadaic acid, an inhibitor of protein phosphatases, 1 and 2A, correlates with the induction of AP1. J Biol Chem. 1992:267:10810-5.

48. Grigoryan T, Wend P, Klaus A, Birchmeier W. Deciphering the function of canonical Wht signals in development and disease: conditional loss- and gain-of-function mutations of $\beta$-catenin in mice. Genes Dev. 2008:22:2308-41. 